\title{
High-sensitivity detection of clinically significant red blood cell antibodies by the column agglutination technique
}

\author{
Nurdina Charong ${ }^{1, A-F}$, Nateelak Kooltheat ${ }^{1, A-E}$, Thunyaluk Plyduang ${ }^{2, B-D}$ \\ ${ }^{1}$ School of Allied Health Sciences, Walailak University, Nakhon Si Thammarat, Thailand \\ ${ }^{2}$ The Centre for Scientific and Technological Equipment, Walailak University, Nakhon Si Thammarat, Thailand \\ A - research concept and design; $B$ - collection and/or assembly of data; $C$ - data analysis and interpretation; \\ $D$ - writing the article; $E$ - critical revision of the article; $F$ - final approval of the article
}

Address for correspondence

Nurdina Charong

E-mail: nurdina.ch@mail.wu.ac.th

Funding sources

None declared

Conflict of interest

None declared

Received on May 11, 2021

Reviewed on May 29, 2021

Accepted on July 19, 2021

Published online on September 10, 2021

\section{Abstract}

Background. The detection of clinically significant antibodies to red blood cell antigens is important for the selection of compatible blood for patients. The conventional test tube technique (CTT) is commonly used as the gold standard test, but manual testing and visual detection of hemagglutination may produce errors. A more recently developed method, the column agglutination technique (CAT), facilitates ease of testing.

Objectives. To investigate the specificity and sensitivity of the CAT compared to the CTT for the screening of clinically significant antibodies from the Rh blood group.

Materials and methods. Standard antibodies to the Rh blood group, anti-D, -C and -E, were used as examples of clinically significant antibodies in transfusion science. The antibodies were serially diluted by twofold, then reacted with screening cells with different antigen expression. The hemagglutination reaction was investigated using both techniques, and the grades and scores of the reactions were used to analyze the sensitivity and specificity of the CTT and CAT.

Results. The CAT had a better sensitivity than the CTT. The lowest antibody dilution of 1:8192 could be detected using CAT, while a dilution of only 1:2048 could be detected with CTT. However, the CTT and CAT were equal in specificity. The 2 techniques specifically detected all antibodies to the screening cells.

Conclusions. Both the CAT and CTT showed 100\% specificity. However, the CAT exhibited more sensitivity than the CTT, and can be used in substitution of, or in parallel with, the CTT technique for red blood cell phenotyping, antibody screening, identification, and crossmatching.

Key words: column agglutination technique, conventional test tube, antibody screening, Rh antibody, anti-D

Cite as

Charong N, Kooltheat N, Plyduang T. High-sensitivity detection of clinically significant red blood cell antibodies by the column agglutination technique. Adv Clin Exp Med. 2021;30(11):1205-1214. doi:10.17219/acem/140317

DOI

10.17219/acem/140317

Copyright

Copyright by Author(s)

This is an article distributed under the terms of the

Creative Commons Attribution 3.0 Unported (CC BY 3.0)

(https://creativecommons.org/licenses/by/3.0/) 


\section{Background}

Antibody screening in blood banking is used to detect unexpected antibodies to red blood cell antigens other than ABO antibodies, known as clinically significant antibodies. ${ }^{1}$ These unexpected antibodies include minor blood groups antibodies, such as anti-Rh, -MNS, -P, -Lewis, -Kell, -Kidd, -Duffy, and -Diego. ${ }^{2}$ According to the AABB standard for Blood Bank and Transfusion Service, antibody screening is performed in 2 situations. First, it is used to screen donor blood in order to prevent the passing of unexpected antibodies to patients and subsequent hemolytic transfusion reactions (HTRs). Second, antibody screening is used pre-transfusion to test a transfusion recipient's sample. ${ }^{3}$ Positive antibody screening results in a donor's plasma lead to the rejection of the plasma unit and, if the transfusion recipient has unexpected antibodies, antibody identification and confirmation tests are necessary to select the antigen negative blood donor for compatibility testing. ${ }^{4}$

Unexpected antibodies can be divided into 2 types according to the source of immunization. Alloantibodies are produced by patient's immunization by foreign red blood cell antigens encountered through blood transfusion or pregnancy. ${ }^{5}$ Autoantibodies are produced after a patient's immune system loses tolerance for self-antigens, which can occur with age and is associated with several diseases. ${ }^{6}$ Depending upon how immunization occurs, unexpected antibodies can also be divided into immune type and naturally occurring antibodies. Immune antibodies, such as anti-Rh, -Kidd or -Duffy, are produced after immunization through blood transfusion or pregnancy. ${ }^{7}$ These antibodies are mostly of the immunoglobulin $\mathrm{G}$ (IgG) type and react best at body temperature $\left(37^{\circ} \mathrm{C}\right)$. Naturally occurring antibodies (e.g., anti-Lewis, -P) are not produced by immunization by red blood cell antigens, but by antigens that are structurally similar to those on red blood cells. ${ }^{8}$ These antibodies are mostly of the IgM type and react best at temperatures below body temperature $\left(4-22^{\circ} \mathrm{C}\right)$. Clinically significant unexpected antibodies are also characterized according to their impact on donor red blood cells after blood transfusion. Highly significant IgG antibodies, such as anti-Rh, react at body temperature, and can strongly induce HTRs and produce hemolytic disease of the fetus and newborn (HDFN). ${ }^{9}$ The other type of unexpected antibodies, IgMs such as anti-I, -M and -N, react at low temperatures and normally cause a positive result in the direct antiglobulin test without hemolysis. ${ }^{10}$

Antibody screening is performed by reacting patient's or donor's serum/plasma with screening cells made from pooled Type $\mathrm{O}$ cells that have different minor red blood cell antigens. ${ }^{11}$ Unexpected antibodies in the sample will bind to the corresponding antigens on the red blood cells and directly cause hemagglutination. For the IgG class of unexpected antibodies, the addition of secondary antihuman globulins (AHG) is needed to crosslink the nearby sensitized red blood cells.
Different techniques can be used for antibody identification and an ideal technique must be able to detect low levels of clinically significant antibodies, be easy to perform, and have a short turnaround time. The conventional test tube technique (CTT) uses a glass test tube as a container for the reaction. For this test, the serum sample is mixed with screening cells, incubated at room temperature $\left(20-22^{\circ} \mathrm{C}\right)$ for the detection of IgM antibodies, and then incubated at body temperature $\left(37^{\circ} \mathrm{C}\right)$ with $\mathrm{AHG}$ for the detection of IgG antibodies. The CTT is considered the gold standard for antibody screening, as it is easy to perform and it is cost effective. ${ }^{12}$ However, the grading of hemagglutination by eye and shaking the test tube by hand requires expertise, and false positive and false negatives can occur easily depending on individual techniques. The column agglutination technique (CAT) is an alternative method for antibody screening that uses a microcolumn gel card as a container for the reaction. For this technique, the serum sample and cells are transferred and mixed in a column gel card, incubated at $37^{\circ} \mathrm{C}$, and spun to read the hemagglutination reaction by visual inspection or use of an automated analyzer. The CAT is easier to carry out, more accurate and more precise than the CTT technique. ${ }^{13}$ However, due to increased costs and the need for specific equipment, the CAT method is typically not used by small community hospitals.

\section{Objectives}

The aim of this study is to compare the CTT and CAT in terms of specificity, accuracy and precision for antibody screening. In order to find a suitable technique for the detection of low antibody titers, clinically significant antibodies of the Rh system (anti-D, -C, -E) were investigated.

\section{Materials and methods}

\section{Reagents and equipment}

Standard antibodies for Rh blood groups, anti-D, anti-C and anti-E, and antibody screening cells (lot: 62061) were purchased from the National Blood Centre (Thai Red Cross Society, Bangkok, Thailand). The expression of Rh antigens on the antibody screening red blood cells used in this study is shown in Table 1 . The column agglutination platform and the AHG/LISS gel cards were commercially purchased (Diagnostic Grifols, Barcelona, Spain).

\section{Antibody dilution and antibody screening cells}

Standard antibodies for Rh blood groups (anti-D, anti-C and anti-E) were two-fold diluted up to 1:16384. A total of $500 \mu \mathrm{L}$ of the antibodies were serially diluted with 
Table 1. Expression of Rh antigens on antibody screening red blood cells

\begin{tabular}{|c|c|c|c|c|c|c|}
\hline \multirow{2}{*}{ Screening cells } & \multicolumn{6}{|c|}{$\mathrm{Rh}$} \\
\hline & Wiener & $\mathrm{D}$ & $\mathrm{C}$ & $\mathrm{E}$ & $c$ & $\mathrm{e}$ \\
\hline 01 & R1R1 & + & + & 0 & 0 & + \\
\hline $\mathrm{O} 2$ & R1R2 & + & + & + & + & + \\
\hline
\end{tabular}

an equal volume of $0.9 \% \mathrm{NaCl}$ (normal saline solution (NSS)) using glass test tubes and a mechanical automatic pipette. The types of screening cells used were based upon expression of the Rh blood group antigens on the cell surface (Table 1). For the inspection of anti-D and anti-C, $\mathrm{O} 1$ or $\mathrm{O} 2$ screening cells were used. For the inspection of anti-E, only the $\mathrm{O} 2$ screening cells were used.

\section{Antibody screening by CTT}

The CTT for antibody screening was performed using the indirect antiglobulin test. First, 2 drops of diluted antibody were added to a $10 \times 75 \mathrm{~mm}$ glass test tube. Next, 1 drop of a $3 \%$ screening cells suspension was added, and the solution was mixed and incubated at room temperature for $5 \mathrm{~min}$. The tube was then centrifuged at $3000 \mathrm{rpm}$ for $15 \mathrm{~s}$ and gently shaken for the visual inspection of hemagglutination. The solution was then further incubated at $37^{\circ} \mathrm{C}$ for $30 \mathrm{~min}$, centrifuged for $15 \mathrm{~s}$, and inspected again for hemagglutination. Incubated screening cells were then washed 3 times with NSS and the supernatant was completely discarded. Two drops of AHG (Thai Red Cross Society) were added to the tube to crosslink antibody-sensitized screening cells. The solution was then centrifuged for $15 \mathrm{~s}$ for the inspection of hemagglutination in the AHG phase. The reactions were graded and scored according to the standard AABB guidelines. ${ }^{14}$

\section{Antibody screening by CAT}

The antibody screening using CAT was performed according to the manufacturer's instructions. Using an AHG/LISS microcolumn gel card, $50 \mu \mathrm{L}$ of $1 \%$ screening cells in NSS were added, followed by $25 \mu \mathrm{L}$ of diluted antibody. The solution was then incubated at $37^{\circ} \mathrm{C}$ for $15 \mathrm{~min}$ in a gel card incubator. The gel card was then centrifuged at $900 \times$ g for 9 min using a specific gel card centrifuge. Hemagglutination was visually graded, scored and compared with control.

\section{Statistical analyses}

This study compared the titer of antibodies that can be detected by antibody screening. The antibody titer range was the range of the two-fold diluted antibody. The mean titer was calculated by averaging the reacted antibody titers. The mean reaction score was calculated by averaging the reaction scores of the antibody tests for the screening of D, C or E antigens on screening red blood cells.
The Gaussian distribution of all data sets from the antibody screenings were analyzed with column statistics using the D'Agostino-Pearson omnibus normality test. The antibody titers detected with the CTT and CAT were compared using paired t-tests (parametric, Gaussian distribution) or Wilcoxon matched-pairs signed-rank tests (non-parametric, non-Gaussian distribution). Correlations between the CTT and CAT were analyzed using Pearson's correlations (parametric) or Spearman's correlations (nonparametric). The correlations between the CTT and CAT in the screening of unexpected antibodies were further analyzed with $2^{\text {nd }}$ order polynomial (quadratic) to maximize the goodness of fit of the analyzed data. All statistical analyses were performed with a 95\% confidence interval, $\mathrm{p}=0.05$, using GraphPad Prism v. 6.01 (GraphPad Software, San Diego, USA).

The sensitivity, specificity, accuracy, and precision of the CTT and CAT in the screening of anti-D, anti-C and anti-E were calculated using a confusion matrix. ${ }^{15}$

All methods and results are reported in agreement with the Standards for Quality Improvement Reporting Excellence (SQUIRE) guidelines. ${ }^{16}$

\section{Results}

\section{Specificity, accuracy and precision of CTT and CAT for antibody screening}

The testing of the CTT and CAT for antibody screening was done by reacting diluted antibodies with screening cells. The screening cells expressed different antigens of the $\mathrm{Rh}$ blood group, with $\mathrm{O} 1$ cells expressing only $\mathrm{D}$ and $\mathrm{C}$ antigens and $\mathrm{O} 2$ cells expressing $\mathrm{D}, \mathrm{C}$ and $\mathrm{E}$ antigens (Table 1). The specificity of the antibody screening techniques was analyzed using the reaction between the screening cells and specific antibodies. The CTT and CAT both gave positive results for reactions between the $\mathrm{O} 1$ or $\mathrm{O} 2$ cells and anti-D and anti-C (Tables 2,3). The 2 techniques also gave negative results for the reaction of $\mathrm{O} 1$ and positive results for the reaction of $\mathrm{O} 2$, when testing with anti-E (Table 4). These results show that both the CTT and CAT have equal specificity in the screening of clinically significant antibodies to D, C and E antigens. Due to the use of $\mathrm{E}$ antigen-negative cells in the screening test, the specificity of anti-E was also calculated (Table 5). The precision of the antibody screening results performed by the CAT was consistent when compared to the CTT and showed a high accuracy for all tested antibodies (Table 5). 
Table 2. Reaction gradings and scores of anti-D screening using CTT and CAT

\begin{tabular}{|c|c|c|c|c|c|c|c|c|c|c|c|c|c|c|c|c|c|c|c|c|c|c|c|c|}
\hline \multicolumn{25}{|c|}{ Anti-D } \\
\hline \multirow{4}{*}{$\begin{array}{c}\text { Dilution } \\
\text { (titer } \\
\text { range) }\end{array}$} & \multicolumn{12}{|c|}{$\begin{array}{l}\text { conventional test tube technique } \\
\text { (CTT) }\end{array}$} & \multicolumn{12}{|c|}{$\begin{array}{l}\text { column agglutination technique } \\
\text { (CAT) }\end{array}$} \\
\hline & \multicolumn{6}{|c|}{ reaction grading } & \multicolumn{6}{|c|}{ score } & \multicolumn{6}{|c|}{ reaction grading } & \multicolumn{6}{|c|}{ score } \\
\hline & \multicolumn{3}{|c|}{01} & \multicolumn{3}{|c|}{$\mathrm{O} 2$} & \multicolumn{3}{|c|}{01} & \multicolumn{3}{|c|}{$\mathrm{O} 2$} & \multicolumn{3}{|c|}{01} & \multicolumn{3}{|c|}{$\mathrm{O} 2$} & \multicolumn{3}{|c|}{01} & \multicolumn{3}{|c|}{$\mathrm{O} 2$} \\
\hline & $1^{\text {st }}$ & $2^{\text {nd }}$ & $3^{\text {rd }}$ & $1^{\text {st }}$ & $2^{\text {nd }}$ & $3^{\text {rd }}$ & $1^{\text {st }}$ & $2^{\text {nd }}$ & $3^{\text {rd }}$ & $1^{\text {st }}$ & $2^{\text {nd }}$ & $3^{\text {rd }}$ & $1^{\text {st }}$ & $2^{\text {nd }}$ & $3^{\text {rd }}$ & $1^{\text {st }}$ & $2^{\text {nd }}$ & $3^{\text {rd }}$ & $1^{\text {st }}$ & $2^{\text {nd }}$ & $3^{\text {rd }}$ & $1^{\text {st }}$ & $2^{\text {nd }}$ & $3^{\text {rd }}$ \\
\hline 1:2 & $4+$ & $4+$ & $4+$ & $4+$ & $4+$ & $4+$ & 12 & 12 & 12 & 12 & 12 & 12 & $4+$ & $4+$ & $4+$ & $4+$ & $4+$ & $4+$ & 12 & 12 & 12 & 12 & 12 & 12 \\
\hline 1:4 & $4+$ & $4+$ & $4+$ & $4+$ & $4+$ & $4+$ & 12 & 12 & 12 & 12 & 12 & 12 & $4+$ & $4+$ & $4+$ & $4+$ & $4+$ & $4+$ & 12 & 12 & 12 & 12 & 12 & 12 \\
\hline 1:8 & $4+$ & $4+$ & $4+$ & $4+$ & $4+$ & $4+$ & 12 & 12 & 12 & 12 & 12 & 12 & $4+$ & $4+$ & $4+$ & $4+$ & $4+$ & $4+$ & 12 & 12 & 12 & 12 & 12 & 12 \\
\hline 1:16 & $4+$ & $4+$ & $4+$ & $4+$ & $4+$ & $4+$ & 12 & 12 & 12 & 12 & 12 & 12 & $4+$ & $4+$ & $4+$ & $4+$ & $4+$ & $4+$ & 12 & 12 & 12 & 12 & 12 & 12 \\
\hline 1:32 & $4+$ & $4+$ & $4+$ & $4+$ & $4+$ & $4+$ & 12 & 12 & 12 & 12 & 12 & 12 & $4+$ & $4+$ & $4+$ & $4+$ & $4+$ & $4+$ & 12 & 12 & 12 & 12 & 12 & 12 \\
\hline 1:64 & $3+$ & $4+$ & $3+$ & $3+$ & $4+$ & $3+$ & 10 & 12 & 10 & 10 & 12 & 10 & $4+$ & $4+$ & $4+$ & $4+$ & $4+$ & $4+$ & 12 & 12 & 12 & 12 & 12 & 12 \\
\hline 1:128 & $2+$ & $2+$ & $2+$ & $3+$ & $4+$ & $3+$ & 8 & 8 & 8 & 10 & 12 & 10 & $4+$ & $4+$ & $4+$ & $4+$ & $4+$ & $4+$ & 12 & 12 & 12 & 12 & 12 & 12 \\
\hline 1:256 & $2+$ & $2+$ & $2+$ & $2+$ & $4+$ & $3+$ & 8 & 8 & 8 & 8 & 12 & 10 & $4+$ & $4+$ & $4+$ & $4+$ & $4+$ & $4+$ & 12 & 12 & 12 & 12 & 12 & 12 \\
\hline 1:512 & $2+$ & $1+$ & $2+$ & $2+$ & $2+$ & $2+$ & 8 & 5 & 8 & 8 & 8 & 8 & $4+$ & $4+$ & $4+$ & $4+$ & $4+$ & $4+$ & 12 & 12 & 12 & 12 & 12 & 12 \\
\hline $1: 1024$ & $2+$ & $1+$ & $2+$ & $1+$ & $1+$ & $1+$ & 8 & 5 & 8 & 5 & 5 & 5 & $3+$ & $4+$ & $3+$ & $3+$ & $4+$ & $4+$ & 10 & 12 & 10 & 10 & 12 & 12 \\
\hline 1:2048 & $1+$ & $1+$ & $1+$ & $1+$ & $1+$ & $1+$ & 5 & 5 & 5 & 5 & 5 & 5 & $3+$ & $3+$ & $3+$ & $3+$ & $3+$ & $3+$ & 10 & 10 & 10 & 10 & 10 & 10 \\
\hline 1:4096 & 0 & 0 & 0 & 0 & 0 & 0 & 2 & 2 & 2 & 2 & 2 & 2 & $2+$ & $2+$ & $2+$ & $2+$ & $2+$ & $2+$ & 8 & 8 & 8 & 8 & 8 & 8 \\
\hline 1:8192 & 0 & 0 & 0 & 0 & 0 & 0 & 2 & 2 & 2 & 2 & 2 & 2 & $1+$ & $1+$ & $1+$ & $1+$ & $1+$ & $1+$ & 5 & 5 & 5 & 5 & 5 & 5 \\
\hline 1:16384 & 0 & 0 & 0 & 0 & 0 & 0 & 2 & 2 & 2 & 2 & 2 & 2 & 0 & 0 & 0 & 0 & 0 & 0 & 2 & 2 & 2 & 2 & 2 & 2 \\
\hline \multicolumn{7}{|l|}{ Sum } & 113 & 109 & 113 & 112 & 120 & 114 & & & & Im & & & 143 & 145 & 143 & 143 & 145 & 145 \\
\hline \multicolumn{7}{|l|}{ Average } & \multicolumn{3}{|c|}{$111.7 \pm 2.3$} & \multicolumn{3}{|c|}{$115.4 \pm 4.2$} & \multicolumn{6}{|c|}{ Average } & & $3.7=$ & & \multicolumn{3}{|c|}{$144.3 \pm 1.2$} \\
\hline \multicolumn{7}{|c|}{ Total (mean reaction score) } & \multicolumn{6}{|c|}{$227.0 \pm 2.0$} & \multicolumn{6}{|c|}{ Total (mean reaction score) } & \multicolumn{6}{|c|}{$288.0 \pm 2.0$} \\
\hline
\end{tabular}

Table 3. Reaction gradings and scores of anti-C screening using CTT and CAT

\begin{tabular}{|c|c|c|c|c|c|c|c|c|c|c|c|c|c|c|c|c|c|c|c|c|c|c|c|c|}
\hline \multicolumn{25}{|c|}{ Anti-C } \\
\hline \multirow{4}{*}{$\begin{array}{c}\text { Dilution } \\
\text { (titer } \\
\text { range) }\end{array}$} & \multicolumn{12}{|c|}{$\begin{array}{l}\text { conventional test tube technique } \\
\text { (CTT) }\end{array}$} & \multicolumn{12}{|c|}{$\begin{array}{l}\text { column agglutination technique } \\
\text { (CAT) }\end{array}$} \\
\hline & \multicolumn{6}{|c|}{ reaction grading } & \multicolumn{6}{|c|}{ score } & \multicolumn{6}{|c|}{ reaction grading } & \multicolumn{6}{|c|}{ score } \\
\hline & \multicolumn{3}{|c|}{$\overline{01}$} & \multicolumn{3}{|c|}{$\overline{\mathrm{O} 2}$} & \multicolumn{3}{|c|}{01} & \multicolumn{3}{|c|}{$\overline{\mathrm{O} 2}$} & \multicolumn{3}{|c|}{01} & \multicolumn{3}{|c|}{$\overline{\mathrm{O} 2}$} & \multicolumn{3}{|c|}{01} & \multicolumn{3}{|c|}{$\overline{\mathrm{O} 2}$} \\
\hline & $1^{\text {st }}$ & $2^{\text {nd }}$ & $3^{\text {rd }}$ & $1^{\text {st }}$ & $2^{\text {nd }}$ & $3^{\text {rd }}$ & $1^{\text {st }}$ & $2^{\text {nd }}$ & $3^{\text {rd }}$ & $1^{\text {st }}$ & $2^{\text {nd }}$ & $3^{\text {rd }}$ & $1^{\text {st }}$ & $2^{\text {nd }}$ & $3^{\text {rd }}$ & $1^{\text {st }}$ & $2^{\text {nd }}$ & $3^{\text {rd }}$ & $1^{\text {st }}$ & $2^{\text {nd }}$ & $3^{\text {rd }}$ & $1^{\text {st }}$ & $2^{\text {nd }}$ & $3^{\text {rd }}$ \\
\hline $1: 2$ & $4+$ & $4+$ & $4+$ & $4+$ & $4+$ & $4+$ & 12 & 12 & 12 & 12 & 12 & 12 & $4+$ & $4+$ & $4+$ & $4+$ & $4+$ & $4+$ & 12 & 12 & 12 & 12 & 12 & 12 \\
\hline $1: 4$ & $4+$ & $4+$ & $4+$ & $4+$ & $4+$ & $4+$ & 12 & 12 & 12 & 12 & 12 & 12 & $4+$ & $4+$ & $4+$ & $4+$ & $4+$ & $4+$ & 12 & 12 & 12 & 12 & 12 & 12 \\
\hline 1:8 & $4+$ & $4+$ & $4+$ & $4+$ & $4+$ & $4+$ & 12 & 12 & 12 & 12 & 12 & 12 & $4+$ & $4+$ & $4+$ & $4+$ & $4+$ & $4+$ & 12 & 12 & 2 & 12 & 12 & 12 \\
\hline 1:16 & $4+$ & $4+$ & $4+$ & $4+$ & $4+$ & $4+$ & 12 & 12 & 12 & 12 & 12 & 12 & $4+$ & $4+$ & $4+$ & $4+$ & $4+$ & $4+$ & 12 & 12 & 12 & 12 & 12 & 12 \\
\hline $1: 32$ & $4+$ & $3+$ & $3+$ & $2+$ & $2+$ & $2+$ & 12 & 10 & 10 & 8 & 8 & 8 & $4+$ & $4+$ & $4+$ & $4+$ & $3+$ & $4+$ & 12 & 12 & 12 & 12 & 10 & 12 \\
\hline 1:64 & $3+$ & $4+$ & $3+$ & $2+$ & $1+$ & $2+$ & 10 & 12 & 10 & 10 & 5 & 8 & $4+$ & $4+$ & $4+$ & $4+$ & $3+$ & $4+$ & 12 & 12 & 12 & 12 & 10 & 12 \\
\hline $1: 128$ & $2+$ & $2+$ & $2+$ & $1+$ & $1+$ & $1+$ & 8 & 8 & 8 & 5 & 5 & 5 & $4+$ & $4+$ & $4+$ & $2+$ & $3+$ & $2+$ & 12 & 12 & 12 & 8 & 10 & 8 \\
\hline 1:256 & $2+$ & $2+$ & $1+$ & $1+$ & $1+$ & $1+$ & 8 & 8 & 5 & 5 & 5 & 5 & $3+$ & $4+$ & $3+$ & $1+$ & $2+$ & $2+$ & 10 & 12 & 10 & 5 & 8 & 8 \\
\hline $1: 512$ & $1+$ & $2+$ & $1+$ & w+ & $1+$ & 0 & 5 & 8 & 5 & 4 & 5 & 2 & $2+$ & $3+$ & $3+$ & 0 & $1+$ & 0 & 8 & 10 & 10 & 2 & 5 & 2 \\
\hline 1:1024 & w+ & $1+$ & w+ & w+ & 0 & 0 & 4 & 5 & 4 & 4 & 2 & 2 & $1+$ & $2+$ & $2+$ & 0 & $1+$ & 0 & 5 & 8 & 8 & 2 & 5 & 5 \\
\hline 1:2048 & 0 & 0 & 0 & 0 & 0 & 0 & 2 & 2 & 2 & 2 & 2 & 2 & 0 & 0 & 0 & 0 & 0 & 0 & 2 & 2 & 2 & 2 & 2 & 2 \\
\hline 1:4096 & 0 & 0 & 0 & 0 & 0 & 0 & 2 & 2 & 2 & 2 & 2 & 2 & 0 & 0 & 0 & 0 & 0 & 0 & 2 & 2 & 2 & 2 & 2 & 2 \\
\hline 1:8192 & 0 & 0 & 0 & 0 & 0 & 0 & 2 & 2 & 2 & 2 & 2 & 2 & 0 & 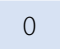 & 0 & 0 & 0 & 0 & 2 & 2 & 2 & 2 & 2 & 2 \\
\hline $1: 16384$ & 0 & 0 & 0 & 0 & 0 & 0 & 2 & 2 & 2 & 2 & 2 & 2 & 0 & 0 & 0 & 0 & 0 & 0 & 2 & 2 & 2 & 2 & 2 & 2 \\
\hline \multicolumn{7}{|l|}{ Sum } & 103 & 107 & 98 & 92 & 86 & 86 & \multicolumn{6}{|c|}{ Sum } & 115 & 122 & 120 & 97 & 104 & 103 \\
\hline \multicolumn{7}{|l|}{ Average } & & $2.7 \pm 4$ & & \multicolumn{3}{|c|}{$88.0 \pm 3.5$} & \multicolumn{6}{|c|}{ Average } & & $9.0 \pm 3$ & & \multicolumn{3}{|c|}{$101.3 \pm 3.8$} \\
\hline \multicolumn{7}{|c|}{ Total (mean reaction score) } & \multicolumn{6}{|c|}{$190.7 \pm 5.7$} & \multicolumn{6}{|c|}{ Total (mean reaction score) } & \multicolumn{6}{|c|}{$220.3 \pm 7.4$} \\
\hline
\end{tabular}


Table 4. Reaction gradings and scores of anti-E screening using CTT and CAT

\begin{tabular}{|c|c|c|c|c|c|c|c|c|c|c|c|c|c|c|c|c|c|c|c|c|c|c|c|c|}
\hline \multicolumn{25}{|c|}{ Anti-E } \\
\hline \multirow{4}{*}{$\begin{array}{c}\text { Dilution } \\
\text { (titer } \\
\text { range) }\end{array}$} & \multicolumn{12}{|c|}{$\begin{array}{l}\text { conventional test tube technique } \\
\text { (CTT) }\end{array}$} & \multicolumn{12}{|c|}{$\begin{array}{l}\text { column agglutination technique } \\
\text { (CAT) }\end{array}$} \\
\hline & \multicolumn{6}{|c|}{ reaction grading } & \multicolumn{6}{|c|}{ score } & \multicolumn{6}{|c|}{ reaction grading } & \multicolumn{6}{|c|}{ score } \\
\hline & \multicolumn{3}{|c|}{01} & \multicolumn{3}{|c|}{$\mathrm{O} 2$} & \multicolumn{3}{|c|}{01} & \multicolumn{3}{|c|}{$\mathrm{O} 2$} & \multicolumn{3}{|c|}{01} & \multicolumn{3}{|c|}{$\mathrm{O} 2$} & \multicolumn{3}{|c|}{$\overline{01}$} & \multicolumn{3}{|c|}{$\overline{\mathrm{O} 2}$} \\
\hline & $1^{\text {st }}$ & $2^{\text {nd }}$ & $3^{\text {rd }}$ & $1^{\text {st }}$ & $2^{\text {nd }}$ & $3^{\text {rd }}$ & $1^{\text {st }}$ & $2^{\text {nd }}$ & $3^{\text {rd }}$ & $1^{\text {st }}$ & $2^{\text {nd }}$ & $3^{\text {rd }}$ & $1^{\text {st }}$ & $2^{\text {nd }}$ & $3^{\text {rd }}$ & $1^{\text {st }}$ & $2^{\text {nd }}$ & $3^{\text {rd }}$ & $1^{\text {st }}$ & $2^{\text {nd }}$ & $3^{\text {rd }}$ & $1^{\text {st }}$ & $2^{\text {nd }}$ & $3^{\text {rd }}$ \\
\hline $1: 2$ & 0 & 0 & 0 & $4+$ & $4+$ & $4+$ & 2 & 2 & 2 & 12 & 12 & 12 & 0 & 0 & 0 & $4+$ & $4+$ & $4+$ & 2 & 2 & 2 & 12 & 12 & 12 \\
\hline $1: 4$ & 0 & 0 & 0 & $4+$ & $4+$ & $4+$ & 2 & 2 & 2 & 12 & 12 & 12 & 0 & 0 & 0 & $4+$ & $4+$ & $4+$ & 2 & 2 & 2 & 12 & 12 & 12 \\
\hline $1: 8$ & 0 & 0 & 0 & $4+$ & $4+$ & $4+$ & 2 & 2 & 2 & 12 & 12 & 12 & 0 & 0 & 0 & $4+$ & $4+$ & $4+$ & 2 & 2 & 2 & 12 & 12 & 12 \\
\hline 1:16 & 0 & 0 & 0 & $4+$ & $4+$ & $4+$ & 2 & 2 & 2 & 12 & 12 & 12 & 0 & 0 & 0 & $4+$ & $4+$ & $4+$ & 2 & 2 & 2 & 12 & 12 & 12 \\
\hline 1:32 & 0 & 0 & 0 & $4+$ & $4+$ & $3+$ & 2 & 2 & 2 & 12 & 12 & 10 & 0 & 0 & 0 & $4+$ & $4+$ & $4+$ & 2 & 2 & 2 & 12 & 12 & 12 \\
\hline $1: 64$ & 0 & 0 & 0 & $4+$ & $3+$ & $3+$ & 2 & 2 & 2 & 12 & 10 & 10 & 0 & 0 & 0 & $4+$ & $4+$ & $3+$ & 2 & 2 & 2 & 12 & 12 & 10 \\
\hline 1:128 & 0 & 0 & 0 & $3+$ & $2+$ & $2+$ & 2 & 2 & 2 & 10 & 8 & 8 & 0 & 0 & 0 & $3+$ & $4+$ & $3+$ & 2 & 2 & 2 & 10 & 12 & 10 \\
\hline 1:256 & 0 & 0 & 0 & $2+$ & $2+$ & $2+$ & 2 & 2 & 2 & 8 & 8 & 8 & 0 & 0 & 0 & $3+$ & $4+$ & $3+$ & 2 & 2 & 2 & 10 & 12 & 10 \\
\hline 1:512 & 0 & 0 & 0 & $1+$ & $2+$ & $2+$ & 2 & 2 & 2 & 5 & 8 & 8 & 0 & 0 & 0 & $2+$ & $3+$ & $2+$ & 2 & 2 & 2 & 8 & 10 & 18 \\
\hline $1: 1024$ & 0 & 0 & 0 & $1+$ & $2+$ & $1+$ & 2 & 2 & 2 & 5 & 8 & 5 & 0 & 0 & 0 & $1+$ & $2+$ & $1+$ & 2 & 2 & 2 & 5 & 8 & 5 \\
\hline $1: 2048$ & 0 & 0 & 0 & w+ & $1+$ & $1+$ & 2 & 2 & 2 & 4 & 5 & 5 & 0 & 0 & 0 & $1+$ & $1+$ & $1+$ & 2 & 2 & 2 & 5 & 5 & 5 \\
\hline 1:4096 & 0 & 0 & 0 & 0 & 0 & 0 & 2 & 2 & 2 & 2 & 2 & 2 & 0 & 0 & 0 & $1+$ & $1+$ & $1+$ & 2 & 2 & 2 & 5 & 5 & 5 \\
\hline 1:8192 & 0 & 0 & 0 & 0 & 0 & 0 & 2 & 2 & 2 & 2 & 2 & 2 & 0 & 0 & 0 & 0 & 0 & 0 & 2 & 2 & 2 & 2 & 2 & 2 \\
\hline 1:16384 & 0 & 0 & 0 & 0 & 0 & 0 & 2 & 2 & 2 & 2 & 2 & 2 & 0 & 0 & 0 & 0 & 0 & 0 & 2 & 2 & 2 & 2 & 2 & 2 \\
\hline \multicolumn{7}{|l|}{ Sum } & 28 & 28 & 28 & 110 & 113 & 108 & \multicolumn{6}{|c|}{ Sum } & 28 & 28 & 28 & 119 & 128 & 127 \\
\hline \multicolumn{7}{|l|}{ Average } & \multicolumn{3}{|c|}{$28.0 \pm 0.0$} & \multicolumn{3}{|c|}{$115.3 \pm 2.5$} & \multicolumn{6}{|c|}{ Average } & \multicolumn{3}{|c|}{$28.0 \pm 0.0$} & \multicolumn{3}{|c|}{$124.7 \pm 4.9$} \\
\hline \multicolumn{7}{|c|}{ Total (mean reaction score) } & \multicolumn{6}{|c|}{$138.3 \pm 2.5$} & & otal (m & ean $r$ & eactio & n scor & & & & 152.7 & \pm 4.9 & & \\
\hline
\end{tabular}

w+ - weakly positive.

Table 5. Sensitivity, specificity, accuracy, and precision of CTT and CAT in the screening of anti-D, anti-C and anti-E

\begin{tabular}{|c|c|c|c|c|c|c|}
\hline \multirow[t]{2}{*}{ Measure } & \multicolumn{3}{|c|}{ Conventional test tube technique (CTT) } & \multicolumn{3}{|c|}{$\begin{array}{l}\text { Column agglutination technique } \\
\text { (CAT) }\end{array}$} \\
\hline & anti-D & anti-C & anti-E & anti-D & anti-C & anti-E \\
\hline Sensitivity & 0.7857 & 0.6786 & 0.7857 & 0.9286 & 0.6667 & 0.8571 \\
\hline Specificity & - & - & 1 & - & - & 1 \\
\hline Accuracy & 0.8757 & 0.6786 & 0.8929 & 0.9286 & 0.6667 & 0.9286 \\
\hline Precision & 1 & 1 & 1 & 1 & 1 & 1 \\
\hline Matthews correlation coefficient & - & - & 0.8044 & - & - & 0.866 \\
\hline
\end{tabular}

\section{Sensitivity of CTT and CAT for antibody screening}

The screening of antibodies using CTT and CAT was performed according to the AABB standard protocols. ${ }^{4}$ The reaction of hemagglutination was visually graded as negative $(0)$, weakly positive $(\mathrm{w}+)$ or positive $(1+, 2+$, $3+, 4+)$. The same reaction was also scored as negative (2), weakly positive (4) or positive $(5,8,10,12)$.

The CTT detected anti-D, anti-C and anti-E at the lowest dilutions of 1:2048, 1:1024 and 1:2048, respectively. The reaction scores for the lowest dilutions of anti-D, anti-C and anti-E were 5, 4 and 4, respectively. The total scores for hemagglutination for anti-D, anti- $\mathrm{C}$ and anti-E were
$227.0 \pm 2.0,190.70 \pm 5.70$ and $138.30 \pm 2.50$, respectively (Tables 2-4).

The CAT detected anti-D, anti-C and anti-E at the lowest dilutions of 1:8192, 1:1024 and 1:4096, respectively. Reaction scores for the lowest dilutions of anti-D, anti-C and anti-E were all at 5 . The total scores for hemagglutination for anti-D, anti-C and anti-E were $288.0 \pm 2.0,220.3 \pm 7.4$ and $152.7 \pm 4.9$, respectively (Tables $2-4$ ).

Column statistics were calculated and the Gaussian distribution of all data sets was tested. Data from the anti-D screening with CTT, and the anti-E screening with CTT and CAT, were normally distributed (Table S1). Data from the anti-D screening with CAT, and the anti-C screening with CTT and CAT, were not normally distributed 
Table S1. Statistical analyses of reaction score in screening of anti-D, anti-C and anti-E using conventional tube test technique (CTT) and column agglutination technique (CAT)

\begin{tabular}{|c|c|c|c|c|c|c|}
\hline \multirow{2}{*}{ Parameter } & \multicolumn{2}{|c|}{ Scrn. of anti-D } & \multicolumn{2}{|c|}{ Scrn. of anti-C } & \multicolumn{2}{|c|}{ Scrn. of anti-E } \\
\hline & CTT & CAT & CTT & CAT & CTT & CAT \\
\hline Mean & 8.107 & 10.29 & 6.810 & 7.869 & 7.881 & 8.905 \\
\hline Standard deviation & 4.029 & 3.148 & 4.186 & 4.389 & 3.986 & 3.960 \\
\hline Standard error of mean & 1.077 & 0.8414 & 1.119 & 1.173 & 1.065 & 1.058 \\
\hline Lower $95 \% \mathrm{Cl}$ of mean & 5.781 & 8.468 & 4.392 & 5.335 & 5.579 & 6.618 \\
\hline Upper $95 \% \mathrm{Cl}$ of mean & 10.43 & 12.10 & 9.227 & 10.40 & 10.18 & 11.19 \\
\hline \multicolumn{7}{|c|}{ D'Agostino-Pearson omnibus normality test } \\
\hline $\mathrm{K} 2$ & 3.087 & 13.12 & 6.095 & 6.398 & 3.269 & 3.136 \\
\hline$p$-value & 0.2136 & 0.0014 & 0.0475 & 0.0408 & 0.1950 & 0.2085 \\
\hline Passed normality test? $($ alpha $=0.05)$ & yes & no & no & no & yes & yes \\
\hline p-value summary & ns & ** & * & * & ns & ns \\
\hline Coefficient of variation & $49.69 \%$ & $30.61 \%$ & $61.48 \%$ & $55.77 \%$ & $50.58 \%$ & $44.47 \%$ \\
\hline
\end{tabular}

${ }^{*} p<0.05 ;{ }^{* *} p<0.01 ;$ ns - not significant; Scrn. - screening.

(Table S1). Thus, parametric tests were used for data sets from the anti-E screenings, while non-parametric tests were applied to data sets from anti-D and anti-C screenings.

The results indicated that CAT had a significantly higher sensitivity than CTT (0.0313 compared to 0.0285 , respectively, $\mathrm{p}=0.0078$; Fig. 1A-Fig. 3A).

\section{Titer range, mean titer and mean reaction scores of antibodies detected using CTT and CAT}

The sensitivity of the CTT and CAT was compared by examining the mean reaction scores, titer ranges and the mean titers of detectable antibodies. The CTT had mean reaction scores for the screening of anti-D, anti-C and anti-E of $227.0 \pm 2.0,190.70 \pm 5.70$ and $138.30 \pm 2.50$, respectively. The antibody titer ranges detected using CTT for anti-D, anti-C and anti-E were all at 0-16387 (Tables 2-4). The CAT had mean reaction scores in the screening of anti-D, anti- $\mathrm{C}$ and anti-E of $288.0 \pm 2.0$, $220.3 \pm 7.4$ and $152.7 \pm 4.9$, respectively. The antibody titer ranges detected with CTT for anti-D, anti-C and anti-E were all at $0-16387$ (Tables 2-4). The column agglutination technique had a better sensitivity in antibody screening than CAT (Fig. 1B-Fig. 3B).
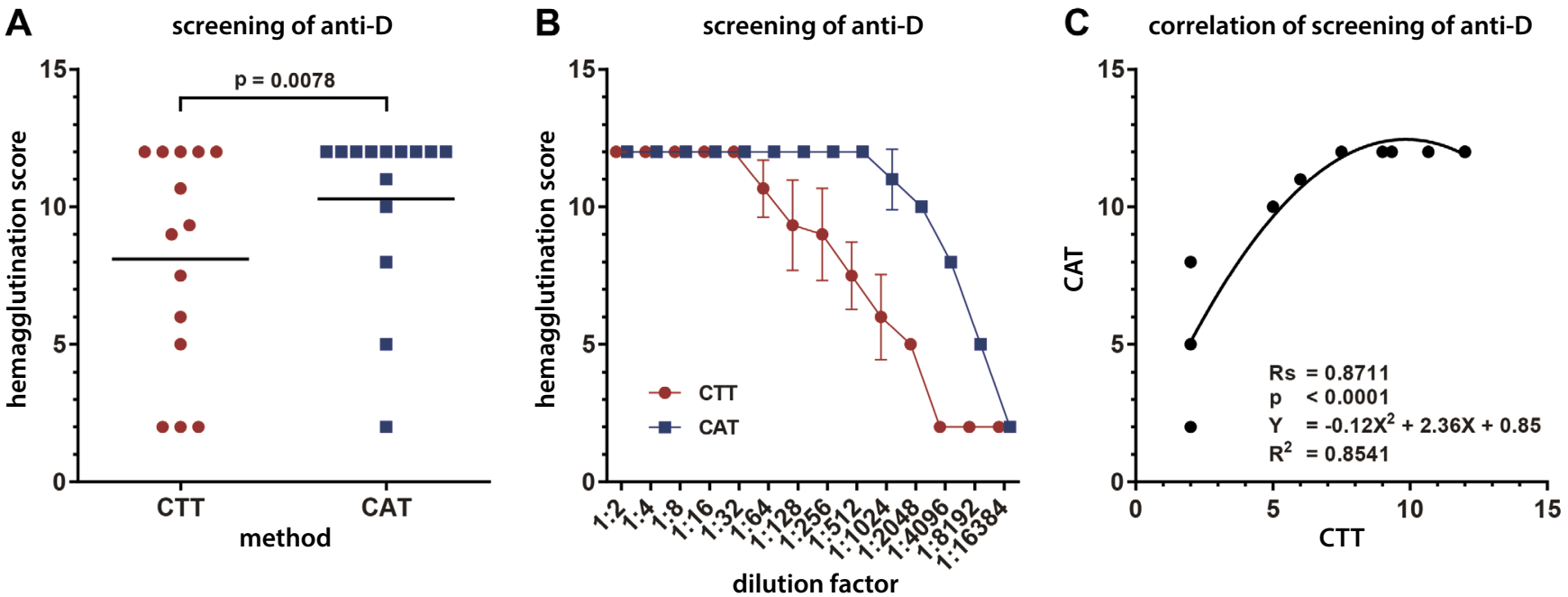

Fig. 1. Sensitivity comparison of the conventional test tube (CTT) and column agglutination techniques (CAT) in the screening of anti-D. Anti-D was two-fold diluted from 1:2 to 1:16348 and then screened with $\mathrm{O} 1$ and $\mathrm{O} 2$ cells using CTT and CAT. The hemagglutination of each reaction was scored. A. Sensitivity of CTT and CAT was compared with Wilcoxon matched-pairs signed-rank test; B. Antibody titers ranging from 1-16384 for anti-D detection using CTT and CAT were used; C. Correlation between CTT and CAT was analyzed with Spearman's correlation and non-linear curve fitting 
A

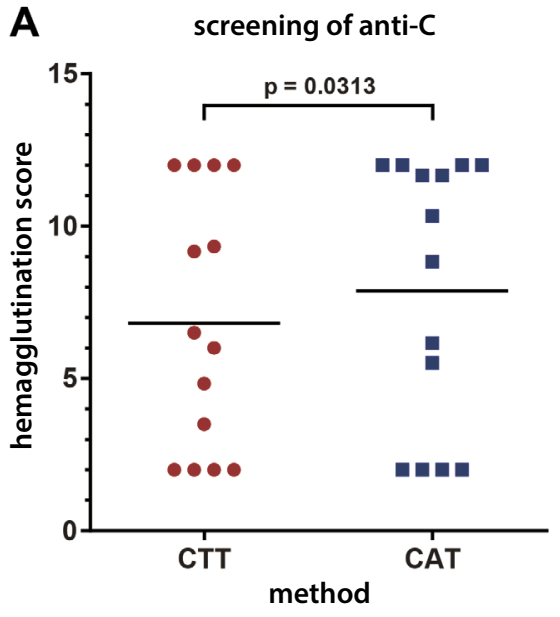

B

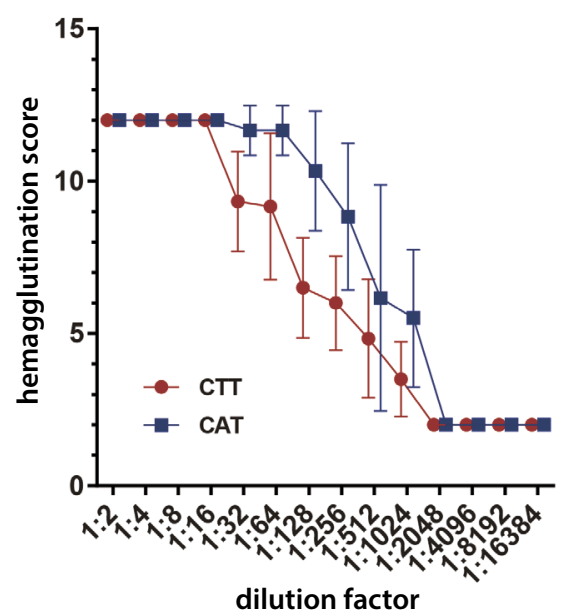

C correlation of screening of anti- $\mathrm{C}$

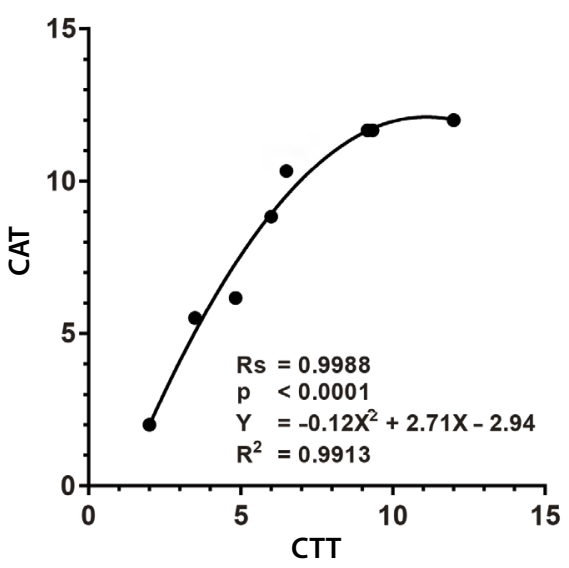

Fig. 2. Sensitivity comparison of the conventional test tube (CTT) and column agglutination techniques (CAT) in the screening of anti-C. Anti-C was two-fold diluted from 1:2 to 1:16348 then screened with $\mathrm{O} 1$ and $\mathrm{O} 2$ cells using CTT and CAT. The hemagglutination of each reaction was scored. A. Sensitivity of CTT and CAT was compared with Wilcoxon matched-pairs signed-rank test; B. Antibody titers ranging from 1 to 16384 for anti-C detection using CTT and CAT were used; C. Correlation between CTT and CAT was analyzed with Spearman's correlation and non-linear curve fitting

Rs - Spearman's correlation coefficient; $Y$ - correlation curve in screening of anti-C; $R^{2}$ - coefficient of determination.

A

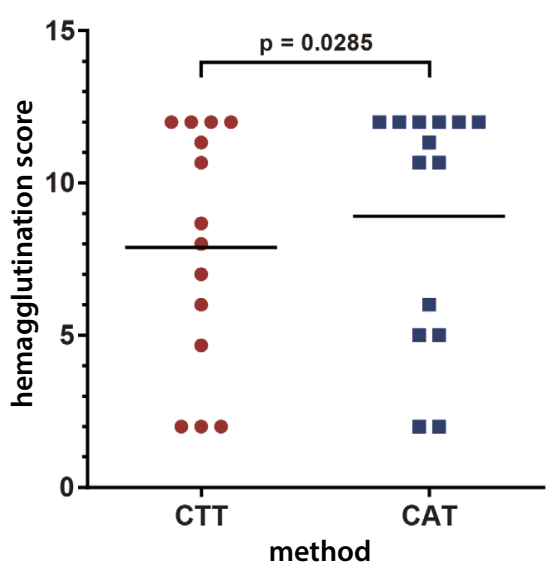

B

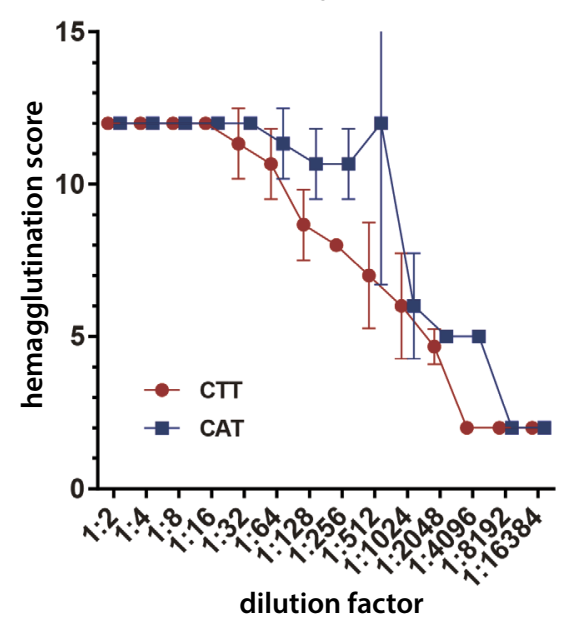

C correlation of screening of anti-E

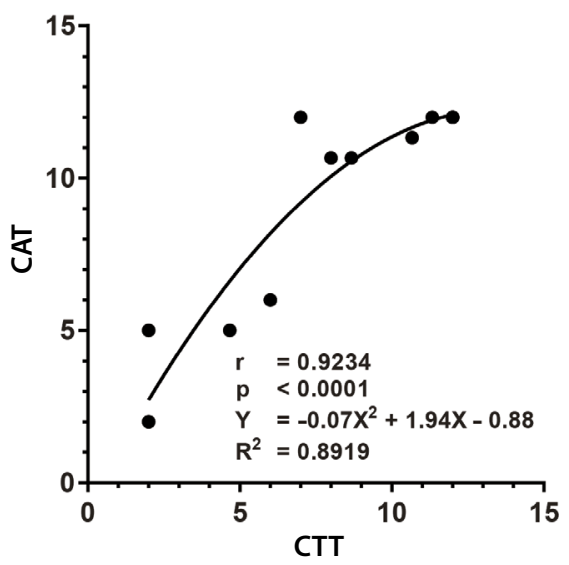

Fig. 3. Sensitivity comparison of conventional test tube (CTT) and column agglutination techniques (CAT) in the screening of anti-E. Anti-E was two-fold diluted from 1:2 to 1:16348 then screened with $\mathrm{O} 1$ and $\mathrm{O} 2$ cells using CTT and CAT. The hemagglutination of each reaction was scored. A. Sensitivity of CTT and CAT was compared by matched-pairs t-test; B. Antibody titers ranging from 1 to 16384 for anti-E detection using CTT and CAT were used; C. Correlation between CTT and CAT was analyzed with Pearson's correlation and non-linear curve fitting

Rs - Spearman's correlation coefficient; $Y$ - correlation curve in screening of anti-C; $R^{2}$ - coefficient of determination.

\section{Differences between CTT and CAT in screening for unexpected clinically significant $\mathrm{Rh}$ antibodies}

Differences in the screening of anti-D, anti-C and anti-E were observed, as CAT has a higher sensitivity than CTT. The screening of anti-D using CAT was significantly different from screening with CTT, with a p-value of 0.0078 , a Spearman's rank correlation coefficient of 0.8711 and a R-square of 0.8541 (Table 6, Fig. 1C). The screening of anti-C using CAT was significantly different from screening with CTT, with a p-value of 0.0313 , a Spearman's rank correlation coefficient of 0.9988 and a R-square of 0.9913 (Table 6, Fig. 2C). The screening of anti-E using CAT was significantly different from screening with CTT with a p-value of 0.0285 , a Pearson's correlation coefficient of 0.9234 and a R-square of 0.8919 (Table 6, Fig. 3C).

\section{Discussion}

The CTT is considered the gold standard for tests in the blood bank laboratory, including for $\mathrm{ABO}$, Rh typing, antibody screening, antibody identification, and crossmatching. ${ }^{5}$ 
Table 6. Differences between conventional test tube technique (CTT) and column agglutination technique (CAT) in screening of unexpected clinically significant Rh antibodies

\begin{tabular}{|c|c|c|c|}
\hline \multirow{2}{*}{ Parameter } & \multicolumn{3}{|c|}{ Screening of unexpected antibody } \\
\hline & anti-D & anti-C & anti-E \\
\hline \multicolumn{4}{|c|}{ Pared t-test } \\
\hline$p$-value & - & - & 0.0285 \\
\hline t-statistic & - & - & 2.463 \\
\hline Degrees of freedom (df) & - & - & 13 \\
\hline Mean of differences & - & - & -0.5119 \\
\hline 95\% confidence interval & - & - & -0.9608 to -0.06297 \\
\hline R-square & - & - & 0.3182 \\
\hline Pearson correlation coefficient (r) & - & - & 0.9234 \\
\hline p-value & - & - & $<0.0001$ \\
\hline \multicolumn{4}{|c|}{ Wilcoxon matched-pairs signed-rank test } \\
\hline$p$-value & 0.0078 & 0.0313 & - \\
\hline Sum of positive & 0.0 & 0.0 & - \\
\hline Negative ranks & -84.0 & -69.0 & - \\
\hline Sum of signed ranks & -84.0 & -69.0 & - \\
\hline Median of differences & -2.0 & 0.0 & - \\
\hline $98.71 \%$ confidence interval & -5.0 to 0.0 & -2.5 to 0.0 & - \\
\hline Spearman's rank correlation coefficient (Rs) & 0.8711 & 0.9988 & - \\
\hline p-value & $<0.0001$ & $<0.0001$ & - \\
\hline
\end{tabular}

This technique uses only basic equipment, is cost-effective and gives significant results in the reading of the reaction. In addition, antibodies of both the IgM and IgG classes can be detected in a single tube at different temperature phases. However, the CTT technique depends on manual testing and reading by the eyes, which may be subject to human error, especially under a high workload. Moreover, the techniques used for tube shaking to resuspend the red cell pellet for observing agglutination may vary among laboratory staff.

In 1985, Lapierre et al. developed a new technique, CAT, for detection of the reaction between red blood cell antigens and antibodies for red blood cell phenotyping. ${ }^{17}$ This method uses a microcolumn packed with gel particles, specific antibodies, LISS buffer, and Coombs/AHG reagent. The Food and Drug Administration (FDA) issued the first gel technology license in 1994 to Micro Typing System under the product name ID-MTS Card. The implementation of this technique in hospital laboratories and transfusion services within 5 years after the release of the first report indicated widespread satisfaction with the test. ${ }^{18,19}$ The CAT is particularly useful as it can eliminate many of the variables associated with manual testing and provides stable endpoint agglutination. The CAT also minimizes sample preparation as can be performed using an automated analyzer, and the results can be repeatedly verified. ${ }^{5}$

The implementation of the CAT for pretransfusion testing was also reported in a community hospital, and indicated an increase in the overall number of antibodies and clinically significant antibodies detected with CAT, as compared to CTT, without significant differences in non-specific reactions. ${ }^{20}$ Earlier studies also compared the CAT with the CTT in terms of ABO, Rh phenotyping, antibody screening, and crossmatching. A strong correlation between the CAT and CTT results for both anti-A and anti-B IgG titers was reported, but these authors suggested that the relationship between CTT and CAT for the titration of other kinds of antibodies should be further analyzed. ${ }^{21}$ Another study also indicated that CAT results showed a high degree of concordance with the CTT, suggesting that the CAT could be brought into routine use. However, proper training and standardization are needed prior to the use of the CAT. ${ }^{22}$ In addition, the costs associated with the CAT are increased compared to the CTT. In the current study, the cost per test for these 2 methods was calculated for a comparison, based on the estimated number of tests per day (10 tests/day) performed by a blood bank in a general or community hospital (Table 7). Interestingly, the estimated cost for CAT is $100 \%$ higher than that for CTT. Thus, CAT should not be recommended for routine use in the laboratory. However, using the CAT in parallel with the CTT for specific cases, as indicated in Fig. 4, is suggested to reduce the adverse effects of transfusion due to the secondary immunization of alloantibody (e.g., delayed hemolytic transfusion reaction (DHTR)).

Although many previous studies have reported on the advantages of using the CAT for detecting unexpected antibodies in terms of sensitivity and specificity, our study showed gaps in the antibody titers that can be detected with these methods. Frequently detectable antibodies to the Rh blood group antigens, anti-D, anti-C and anti-E, were used as model antibodies, which can be 
Table 7. Cost comparison analysis of antibody screening by conventional tube test technique (CTT) and column agglutination technique (CAT)

\begin{tabular}{|c|c|c|c|c|}
\hline \multirow{2}{*}{$\begin{array}{l}\text { Equipment/ } \\
\text { reagent }\end{array}$} & \multicolumn{2}{|l|}{ CTT } & \multicolumn{2}{|l|}{ CAT } \\
\hline & system & $\begin{array}{c}\text { cost* }^{*} \\
\text { [USD/year] }\end{array}$ & system & $\begin{array}{c}\text { cost* }^{*} \\
\text { [USD/year] }\end{array}$ \\
\hline $\begin{array}{l}\text { Reaction } \\
\text { container }\end{array}$ & $\begin{array}{l}10 \times 75 \text { Glass Test Tube (reusable) } \\
\text { (PYREX VISTA, Corning Inc., Glendale, USA) } \\
\text { (1000 tubes) }\end{array}$ & $\begin{array}{c}380 \\
(0.38 / \text { Tube })\end{array}$ & $\begin{array}{c}\text { AHG/LISS Microcolumn (Disposable) } \\
\text { (8 reactions/card, } 50 \text { cards/box) } \\
\text { (DG Gel Coombs, Grifols, S.A., Barcelona, Spain) } \\
\text { (19 boxes) }\end{array}$ & $\begin{array}{c}5947 \\
(313 / \mathrm{Box})\end{array}$ \\
\hline Screening cell & $\begin{array}{l}\text { O1, } 02 \text { ( } 25 \text { HL/test, } 10 \text { mL/bottle/month) } \\
\text { (National Blood Centre, Bangkok, Thailand) } \\
\text { (12 bottles 01, } 12 \text { bottle O2) }\end{array}$ & $\begin{array}{c}28.8 \\
(1.2 / \text { Bottle })\end{array}$ & $\begin{array}{l}\text { O1, } 02 \text { ( } 25 \text { HL/test, } 10 \text { mL/bottle/month) } \\
\text { (National Blood Centre, Bangkok, Thailand) } \\
\text { (12 bottles 01, } 12 \text { bottle O2) }\end{array}$ & $\begin{array}{c}28.8 \\
(1.2 / \text { bottle })\end{array}$ \\
\hline $\begin{array}{l}\text { Anti-human } \\
\text { globulin reagent }\end{array}$ & $\begin{array}{c}\text { AHG/Coomb's ( } 50 \text { HL/test, } 10 \text { mL/bottle) } \\
\text { (National Blood Centre, Bangkok, Thailand) } \\
\text { ( } 37 \text { bottles) }\end{array}$ & $\begin{array}{c}118.4 \\
(3.2 / \text { Bottle) }\end{array}$ & - & - \\
\hline $\begin{array}{l}\text { Low ionic } \\
\text { strength solution }\end{array}$ & $\begin{array}{c}\text { LISS (100 } \mu \text { L/test, } 10 \text { mL/bottle) } \\
\text { (National Blood Centre, Bangkok, Thailand) } \\
\text { (74 bottles) }\end{array}$ & $\begin{array}{c}236.8 \\
(3.2 / \text { Bottle) }\end{array}$ & - & - \\
\hline Incubator & $\begin{array}{c}\text { Heat Block } \\
\text { (DVT-2B, BioVision Inc., Milpitas, USA) }\end{array}$ & 360 & $\begin{array}{l}\text { Microcolumn Card Incubator } \\
\text { (DG Therm, Grifols, S.A., Barcelona, Spain) }\end{array}$ & 1625 \\
\hline Centrifuge & $\begin{array}{c}\text { Serofuge } \\
\text { (EBA } 280 \text { Serology Decanting Package 5, Andreas } \\
\text { Hettich GmbH \& Co. KG, Tuttlingen, Germany) }\end{array}$ & 4481 & $\begin{array}{c}\text { Microcolumn Centrifuge } \\
\text { (DG Spin, Grifols, S.A., Barcelona, Spain) }\end{array}$ & 5063 \\
\hline $\begin{array}{l}\text { Tube cleaning } \\
\text { cost }\end{array}$ & every week (48 ×10 USD) & 480 & - & - \\
\hline Total & 7300 tests/year & 6085 & 7300 tests/year & 12663.8 \\
\hline Cost & per 1 test & 0.83 & per 1 test & 1.73 \\
\hline \multirow{2}{*}{ Difference } & CTT compared to CAT & \multicolumn{3}{|c|}{$208.12 \%$} \\
\hline & CAT compared to CTT & \multicolumn{3}{|c|}{$48.05 \%$} \\
\hline
\end{tabular}

Cost analyzed from product supplied in Thailand. ${ }^{*} 10$ tests of antibody screening $(\mathrm{O} 1, \mathrm{O} 2)$ per day, 7300 tests for 1 year.

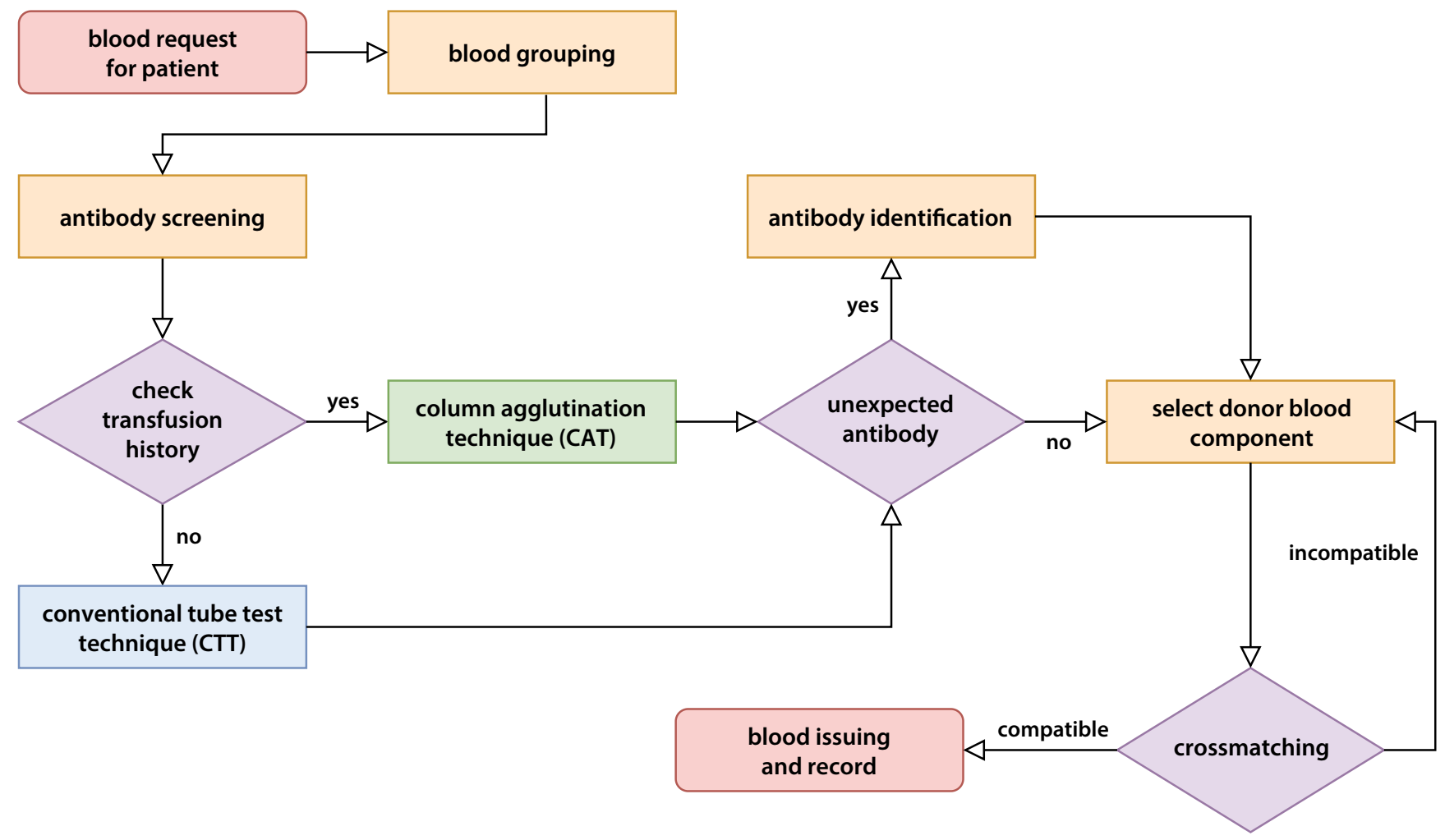

Fig. 4. Suggested guidelines for using column agglutination technique (CAT) in parallel with conventional tube test technique (CTT) in specific case of blood requesting 
applied in the investigation of DHTR and HDFN in real-life situations. While the CAT was significantly comparable to the gold standard technique CTT $(\mathrm{p}<0.01)$, the CAT had a higher sensitivity than the standard CTT, and the mean titer of CAT in the detection of anti-D, anti-C and anti-E was significantly higher than CTT $(\mathrm{p}<0.01)$.

Thus, the use of CAT instead of CTT may be better in detecting lower-titer antibodies, especially for the clinically significant antibodies. In addition, to prevent DHTRs, CAT should be carried out for the detection of low-titer antibodies that have been previously detected in a patient, but where no agglutination appeared with the CTT. Nevertheless, IgM class unexpected antibodies cannot be detected using CAT, as this technique does not have the ability to detect antibodies at room temperature. Therefore, in the case of cold alloantibody detection, the use of CAT in pre-transfusion compatibility testing or crossmatching should be performed in parallel with CTT or using the neutral Gel card. Column agglutination technique has been reported to estimate anti $\mathrm{A} / \mathrm{B}$ titer in an $\mathrm{ABO}$ incompatible kidney transplant, which indicated a sensitivity approx. 2.5-fold higher than CTT. ${ }^{23}$ However, CAT was not suggested to determine a critical titer for anti-D titration, since it showed significantly higher titers than the CTT in all samples studied. ${ }^{24}$

\section{Limitations}

The current study indicates the superior sensitivity of the CAT technique with a wide-gap titer in the detection of commercially available antibodies to the Rh system, compared with the CTT. However, applying the CAT to detect the presence of significant unexpected antibodies in clinical samples is needed to confirm the advantages of the CAT over the CTT. The simpler CAT technique minimizes turnaround time and workload while maintaining standard operation and results, and can easily be performed by blood bank laboratory technologists.

\section{Conclusions}

The CAT can be used in substitution of, or in parallel with, the CTT for the screening of clinically significant antibodies (especially those with a low titer) to avoid secondary immunization of antibodies to minor red blood cell antigens after transfusion.

\section{ORCID iDs}

Nurdina Charong (10 https://orcid.org/0000-0002-4432-2699 Nateelak Kooltheat (1) https://orcid.org/0000-0001-7623-854X Thunyaluk Plyduang (i) https://orcid.org/0000-0002-9523-746X

\section{References}

1. Fung MK, Grossman BJ, Hillyer CD, Westhoff CM, eds. Technical Manual. $18^{\text {th }}$ ed. Bethesda, USA: AABB; 2014:391-396.
2. Giblett ER. Blood group alloantibodies: An assessment of some laboratory practices. Transfusion. 1997;17(4):299-308. doi:10.1046/j.15372995.1977.17477216857.x

3. Nance ST. Red cell antibody detection by serology. ISBT Sci Ser. 2015; 10(Suppl 1):1-4:225-231. doi:10.1111/voxs.12128

4. Walker PS, Hamilton JR. Identification of antibodies to red cell antigens. In: Fung MK, Grossman BJ, Hillyer CD, Westhoff CM, eds. Technical Manual. $18^{\text {th }}$ ed. Bethesda, USA: AABB; 2014:391-421.

5. Avent ND. Transfusion and Transplantation Science. Oxford, UK: Oxford University Press; 2018.

6. Tomer Y, Shoenfeld Y. Ageing and autoantibodies. Autoimmunity. 1988;1(2):141-149. doi:10.3109/08916938809001927

7. Daniels G, Poole J, de Silva M, et al. The clinical significance of blood group antibodies. Transfus Med. 2002;12(5):287-295. doi:10.1046/j. 1365-3148.2002.00399.x

8. Quinley ED. Immunohematology Principles and Practice. Philadelphia, USA: Lippincott Williams and Wilkins; 2011.

9. Urbaniak SJ, Greiss MA. RhD haemolytic disease of the fetus and the newborn. Blood Rev. 2000;14(1):44-61. doi:10.1054/blre.1999.0123

10. Poole J, Daniels G. Blood group antibodies and their significance in transfusion medicine. Transfus Med Rev. 2007;21(1):58-71. doi:10. 1016/j.tmrv.2006.08.003

11. Downes KA, Shulman IA. Antigen and antibody testing. In: Fung MK, Grossman BJ, Hillyer CD, Westhoff CM, eds. Technical Manual. $18^{\text {th }}$ ed. Bethesda, USA: AABB; 2014:367-384.

12. Rumsey $D H$, Ciesielski DJ. New protocols in serologic testing: A review of techniques to meet today's challenges. Immunohematology. 2000; 16(4):131-137. PMID:15373603

13. Langston MM, Procter JL, Cipolone KM, Stroncek DF. Evaluation of the gel system for ABO grouping and D typing. Transfusion. 1999; 39(3):300-305. doi:10.1046/j.1537-2995.1999.39399219288.x

14. Roback JD, Grossman BJ, Harris T, Hillyer CD, eds. Technical Manual. $17^{\text {th }}$ ed. Bethesda,USA: AABB; 2011:873-874.

15. Chicco D, Tötsch N, Jurman $G$. The Matthews correlation coefficient (MCC) is more reliable than balanced accuracy, bookmaker informedness, and markedness in two-class confusion matrix evaluation. BioData Min. 2021;14(1):13. doi:10.1186/s13040-021-00244-z

16. Ogrinc G, Davies L, Goodman D, et al. SQUIRE 2.0 (Standards for Quality Improvement Reporting Excellence): Revised publication guidelines from a detailed consensus process. BMJ Qual Saf. 2016;25:986-992. doi:10.1016/j.jamda.2021.06.006

17. Lapierre $Y$, Rigal $D$, Adam J, et al. The gel test: $A$ new way to detect red cell antigen-antibody reactions. Transfusion. 1990;30(2):109-113. doi:10.1046/j.1537-2995.1990.30290162894.x

18. Cheng G, Chan A, Chun M, et al. Evaluation of the gel test for antibody screening in a tertiary hospital in Hong Kong: Insensitivity for some cold antibodies that are reactive at $37^{\circ} \mathrm{C}$ by conventional indirect antiglobulin tests. Clin Lab Haematol. 1995;17(1):81-84. doi:10.1111/j.1365-2257.1995.tb00323.x

19. Chan HF, Wong $\mathrm{CH}$, Chui L, et al. The impact of a gel system on routine work in a general hospital blood bank. Immunohematology. 1996; 12(1):30-32. PMID:15387759

20. Delaflor-Weiss E, Chizhevsky V. Implementation of gel testing for antibody screening and identification in a community hospital: A 3-year experience. Lab Med. 2005;36(8):489-492. doi:10.1309/ JAP6EC69BAAUG9B3

21. Cheng D, Hao Y. Comparative evaluation of the microcolumn gel card test and the conventional tube test for measurement of titres of immunoglobulin $\mathrm{G}$ antibodies to blood group $\mathrm{A}$ and blood group $\mathrm{B}$. J Int Med Res. 2011;39(3):934-943. doi:10.1177/147323001103900328

22. Bhagwat SN, Sharma JH, Jose J, et al. Comparison between conventional and automated techniques for blood grouping and crossmatching: Experience from a tertiary care centre. J Lab Physicians. 2015;7(2):96-102. doi:10.4103/0974-2727.163130

23. Bhangale $A$, Pathak $A$, Pawar $S$, et al. Comparison of antibody titers using conventional tube technique versus column agglutination technique in $A B O$ blood group incompatible renal transplant. Asian J Transfus Sci. 2017;11(2):131-134. doi:10.4103/0973-6247.214343

24. Novaretti $M C Z$, Jens E, Pagliarini T, et al. Comparison of conventional tube test with diamed gel microcolumn assay for anti-D titration. Clin Lab Haematol. 2003;25(5):311-315. doi:10.1046/j.1365-2257.2003. 00540.x 